\title{
Therapeutic Drug Monitoring of Carbamazepine and Phenytoin: Monotherapy versus Combination Therapy
}

Authors

\section{Dr Rupali Bandagi ${ }^{1}$, Dr Bharti Daswani ${ }^{2}$, Dr Balasaheb Ghongane ${ }^{3}$}

${ }^{1}$ Junior Resident, ${ }^{2}$ Associate Professor, ${ }^{3}$ Professor and Head

Department of Pharmacology, B. J. Government Medical College \& Sassoon General Hospital, Pune,

Maharashtra, India

Corresponding Author

Dr. Rupali Bandagi

Department of Pharmacology, B. J. Government Medical College \& Sassoon General Hospital, Pune,

Maharashtra, India

Phone (or Mobile) No.: +91-9561756529, Email: rrrupali.43@gmail.com

\begin{abstract}
Objectives: To study serum concentration of carbamazepine and phenytoin, when given singly and together. Method: This is a non-interventional, cross-sectional, analytical study done in a tertiary care hospital. Consenting epileptics on stable antiepileptic regime for at least past 2 weeks and taking carbamazepine 200mg $B D$ (group I) or phenytoin 100mg BD (group II) or carbamazepine (200)+phenytoin (100)mg BD (group III) were included. There were 20 patients in each group. Blood samples were collected 12 hours after the last dose and serum was analyzed for carbamazepine, phenytoin concentrations using HPLC method and data was compared using unpaired t-test.

Results: Mean serum carbamazepine concentration in group I was $(7.37 \pm 3.67) \mathrm{mcg} / \mathrm{ml}$ and in group III $(5.65 \pm 2.37) \mathrm{mcg} / \mathrm{ml}(\mathrm{p}=0.09)$. Mean serum phenytoin in group II was $(15.16 \pm 6.48) \mathrm{mcg} / \mathrm{ml}$ and in group III $(12.66 \pm 5.5) \mathrm{mcg} / \mathrm{ml}(p=0.2)$. When used singly carbamazepine showed 30\% subtherapeutic and $20 \%$ toxic concentrations. Whereas in combination group the plasma concentrations of carbamazepine were found to be therapeutic, subtherapeutic and toxic ranges in 55\%, 40\% and 5\% patients, respectively. In phenytoin monotherapy group the concentrations were subtherapeutic in 15\%, therapeutic in $65 \%$ and in toxic range in $20 \%$ patients, whilst in combination group the concentrations obtained were 50\%, 40 and 10\% respectively.

Conclusion: Co-administration of carbamazepine and phenytoin do not affect the serum concentrations of either group significantly. It's possible that Indian population has different therapeutic range of antiepileptic drugs from that given in standard literature. But larger study needs to be done to confirm the results.
\end{abstract}

Keywords: Therapeutic drug monitoring, HPLC, Phenytoin, Carbamazepine.

\section{INTRODUCTION}

Epilepsy is one of the most common serious chronic neurological disorders. It is a disorder of brain which is characterized by an enduring predisposition to generate seizures and by its neurobiological, cognitive, psychological, and social consequences. International League Against 
Epilepsy (ILAE; 1993) defines epilepsy as a condition characterized by recurrent (two or more) epileptic seizures, unprovoked by any immediate identified cause. [Epilepsy_Indian perspective] Antiepileptic medication is the mainstay of treatment of most patients of epilepsy (those without a reversible cause). ${ }^{[1]}$

Therapy for an epileptic patient is almost always multimodal and includes treatment of underlying conditions that cause or contribute to the seizures, avoidance of precipitating factors, suppression of recurrent seizures by prophylactic therapy with antiepileptic medications or surgery, and addressing a variety of psychological and social issues. ${ }^{[2]}$

Anti-epileptic medication is the mainstay of treatment of most patients of epilepsy (those without a reversible cause). Often, antiepileptic treatment will be long-term and can have major effects on quality of life. Seizure classification is an important element in designing the treatment plan, since some antiepileptic drugs have different activities against various seizure types. However, there is considerable overlap between many antiepileptic drugs, such that choice of therapy is often determined more by specific needs of the patient, especially the patient's assessment of sideeffects. ${ }^{[3]}[4]$

Moreover, monotherapy with antiepileptic drugs often fails, requiring polytherapy regimens in an attempt to achieve better seizure control and fewer side effects. Most side effects are mild and doserelated and can often be avoided or minimized by the use of the smallest effective dose. Some examples include mood changes, drowsiness, or unsteadiness in gait. Some antiepileptic medications have idiosyncratic side effects that cannot be predicted by dose. Some examples include drug rashes, liver toxicity (hepatitis), or bone marrow suppression. ${ }^{[5][6]}$

Unfortunately, it is incontestable that these clinical practices of poly- and add-on therapies frequently lead to complex and unpredictable pharmacokinetic and pharmacodynamic interactions, with possible clinical consequences in terms of toxicity or even therapeutic inefficacy. ${ }^{[7][8]}$ All these short comings coupled to fact that the pharmacotherapy with antiepileptic drugs is usually prophylactic and lifelong, and the relationship between dose and drug concentrations is unpredictable, the effectiveness of antiepileptic drugs therapy frequently requires therapeutic drug monitoring procedures. Based on the measurement of drug concentrations in plasma, therapeutic drug monitoring is therefore largely employed with the purpose of avoiding the risks of acute and chronic toxicity ${ }^{[9][10]}$ and to optimize the patient's clinical outcome. Indeed, antiepileptic drugs can be used more safely if the clinician is aware of the concentrations the patient is exposed to and adjusts the dose as necessary ${ }^{[11][12]}$

Carbamazepine (CBZ) and phenytoin (PHT) are first line antiepileptic drugs. Despite the availability of newer antiepileptic drugs, these first line drugs are commonly used because of their efficacy and low cost. However both the drugs have narrow therapeutic index. Hence, monitoring of their serum concentration is essential in most cases. An overlap between phenytoin and carbamazepine toxicity has also been reported. Both are enzyme inducers and thus affect each other's serum concentrations. ${ }^{[13]}$.

\section{METHODS}

This was an observational, cross-sectional, analytical, single center study done in 6 months (June 2016 to November 2016) duration. The study protocol was approved by Institutional Ethics committee. It was conducted in tertiary care hospital in Pune, Maharashtra. Diagnosed epileptics, coming to Neurology OPD in the Medicine Department, who were on regular stable antiepileptic regime for atleast past 2 weeks and taking either carbamazepine $200 \mathrm{mg} \mathrm{BD}$ or phenytoin $100 \mathrm{mg} \mathrm{BD}$ or carbamazepine + phenytoin $(200+100) \mathrm{mg}$ BD, were included. Twenty patients from each category were selected for the study. To minimize batch inconsistency, it was ensured that patients were taking drugs belonging to same batch. Drug details were as follow- Tablet carbamazepine (Ciron drugs \& pharmaceuticals Pvt. Ltd., batch 601013, Mfg. date 01/2016 and Exp. date 12/2017) and Tablet 
phenytoin (Unicure India Ltd., batch PST03-35, Mfg. date 12/2015 and Exp. date 11/2017).

Patients with other concurrent diseases that are known to interact with the above mentioned drugs, patients receiving any drug other than folic acid and calcium supplements were excluded. The study protocol was explained in detail to the patients in their own language. Patients willing to participate in the study were asked to fill and sign an informed consent form. They were asked to maintain a daily drug diary for 2 weeks mentioning time of drug administration and adverse drug effect, if any, to ensure complete adherence to the therapy. Basic information like demographic data, detailed medical history, drug details including batch number, manufacturing date, expiry date, manufacturing company were recorded on a study specific Case Record Form. Patients were examined for adverse effects, if any. Fasting blood samples were collected in the morning 12 hours after the last night dose. Blood was allowed to clot at room temperature. Serum thus obtained on centrifugation was stored at $-20^{\circ} \mathrm{C}$. Chromatographic analysis was carried out using an HPLC system (Hitachi Elite La Chrom). ClinRep® complete kit for antiepileptics in serum was used. All instrumental parts were automatically controlled by Agilent Chem Station software (Agilent Technologies). Temperature was set to $55^{\circ} \mathrm{C}$. Flow rate was set at $1.0 \mathrm{~mL} / \mathrm{min}$. The injection volume was $10 \mathrm{microL}$ and the wavelength of $205 \mathrm{~nm}$ was selected for the detection of both compounds as per instructions in the kit manual.

\section{Method of HPLC}

\section{EXTRACTION}

\begin{tabular}{|c|c|}
\hline $\begin{array}{c}100 \mu 1 \text { sample } \\
\text { (calibrator, control, } \\
\text { patient) }\end{array}$ & $\begin{array}{c}150 \mu 1 \text { Precipitant P } \\
\text { (contains internal standard) }\end{array}$ \\
\hline
\end{tabular}

$\downarrow$ Mix for $30 \mathrm{sec}$ using cyclomixer.

$\downarrow$ Centrifuge for $5 \mathrm{~min}$ at $10000 \mathrm{xg}$ DILUTION

\begin{tabular}{|l|l}
$50 \mu 1$ Supernatant & $50 \mu 1$ Diluting Solution D \\
\hline
\end{tabular}

HPLC analysis

Inject $10 \mu 1$ of diluted supernatant into HPLC system and read at $205 \mathrm{~nm}$
Inject $10 \mu 1$ of diluted supernatant into HPLC system and read at $205 \mathrm{~nm}$

Serum concentration was calculated using internal standard method. Reference Therapeutic serum concentration of phenytoin was taken as 10$20 \mathrm{mcg} / \mathrm{ml}$ and that of carbamazepine $5-12 \mathrm{mcg} / \mathrm{ml}$. $^{[4]}$ Analysis of variance

(ANOVA) was used to comparedemographic characteristics among the three groups of patients. Unpaired t-test was applied to compare serum concentrations of each drug (carbamazepine and phenytoin) in both mono and combination groups. Linear regressionand correlation coefficient were used to test the associations between serum concentrations and seizure frequency.

Table no. 1: Demographic data of patientsin all three groups (mean $\pm \mathrm{SD}$ )

\begin{tabular}{|c|c|c|c|c|}
\hline & & $\begin{array}{c}\text { CBZ } \\
\text { monotherapy }\end{array}$ & $\begin{array}{c}\mathrm{CBZ}+\mathrm{PHT} \\
\text { combitherapy }\end{array}$ & $\begin{array}{c}\text { PHT } \\
\text { monotherapy }\end{array}$ \\
\hline \multicolumn{2}{|l|}{$\mathrm{n}$} & 20 & 20 & 20 \\
\hline \multicolumn{2}{|l|}{$\mathrm{M} / \mathrm{F}$ ratio } & $12 / 8$ & $9 / 11$ & $13 / 7$ \\
\hline \multicolumn{2}{|c|}{ Age (Yrs) } & $35.1 \pm 12.89$ & $35.6 \pm 13.23$ & $34.2 \pm 14.18$ \\
\hline \multicolumn{2}{|c|}{ Weight (Kg) } & $64.6 \pm 15.16$ & $60.1 \pm 13.24$ & $63.85 \pm 12.38$ \\
\hline \multirow[b]{2}{*}{$\begin{array}{l}\text { Dose } \\
(\mathrm{mg} / \mathrm{Kg} / \\
\text { d) }\end{array}$} & CBZ & $6.98 \pm 1.87$ & $6.42 \pm 2.39$ & - \\
\hline & PHT & - & $3.5 \pm 0.86$ & $3.26 \pm 0.72$ \\
\hline
\end{tabular}

\section{RESULTS}

A total of 60 patients were included in study during 6 months data collection period. Out of which $56.66 \%$ were male population and $43.33 \%$ were female population. The clinical details of the patients like age, gender distribution, daily dose of carbamazepine and phenytoin, are shown in Table 1. All three groups were compared using ANOVA test and unpaired t-test. The Baseline characteristics - Age, gender,weight were comparable in all the Groups ( $\mathrm{p}>0.05)$.

Table no. 2: Serum levels of carbamazepine and phenytoin in all three groups (mean \pm SD)

\begin{tabular}{|l|c|c|}
\hline CBZ monotherapy & $\begin{array}{c}\mathrm{CBZ} \\
(\mathrm{mcg} / \mathrm{ml})\end{array}$ & $\begin{array}{c}\mathrm{PHT} \\
(\mathrm{mcg} / \mathrm{ml})\end{array}$ \\
\hline CBZ+PHTcombitherapy & $7.37 \pm 3.67$ & - \\
\hline PHTmonotherapy & $5.65 \pm 2.37$ & $12.66 \pm 5.5$ \\
\hline P Value & - & $15.16 \pm 6.48$ \\
\hline
\end{tabular}

\#Means were not significantly different by analysis of unpaired t-test. 
Table no. 3 Concentration ranges of carbamazepine and phenytoin in all three groups( $\mathrm{N}$ and \%)

\begin{tabular}{|l|c|c|c|c|}
\hline & \multicolumn{2}{|c|}{ CBZ } & \multicolumn{2}{c|}{ PHT } \\
& $\begin{array}{c}\text { Monothera } \\
\text { py }\end{array}$ & $\begin{array}{c}\text { Combithera } \\
\text { py }\end{array}$ & $\begin{array}{c}\text { Monotherap } \\
\text { y }\end{array}$ & $\begin{array}{c}\text { Combitherap } \\
\text { y }\end{array}$ \\
\hline Subtherapeutic & $6(30 \%)$ & $8(40 \%)$ & $3(15 \%)$ & $8(40 \%)$ \\
\hline Therapeutic & $10(50 \%)$ & $11(55 \%)$ & $13(65 \%)$ & $10(50 \%)$ \\
\hline Toxic & $4(20 \%)$ & $1(5 \%)$ & $4(20 \%)$ & $2(10 \%)$ \\
\hline
\end{tabular}

As depicted in table no. 2, mean serum carbamazepine concentration in monotherapy group was $7.37 \pm 3.67 \mathrm{mcg} / \mathrm{ml}$ and in combination therapy group, it was found to be $5.65 \pm 2.37 \mathrm{mcg} / \mathrm{ml}$ $(\mathrm{p}=0.08)$.

Mean serum phenytoinin monotherapy group was $15.16 \pm 6.48 \mathrm{mcg} / \mathrm{ml}$ and in combination therapy group, it was around $12.66 \pm 5.5 \mathrm{mcg} / \mathrm{ml}$ $(p=0.195)$.It was observed that, although the patients receiving carbamazepine + phenytoin tended to have lower carbamazepine and phenytoin levels than the other two groups of patients, the differences did not reach statistical significance.

As shown in Table no.3, when used singly carbamazepine produced therapeutic concentration in $50 \%$ patients while concentrationwas subtherapeutic in $30 \%$ and toxic in $20 \%$ subjects.

Table no. 4: Adverse effects seen in all three groups.

( $\mathrm{N}$ and \%)

\begin{tabular}{|l|c|c|c|}
\hline & $\begin{array}{c}\text { CBZ } \\
\text { monothera } \\
\text { py }\end{array}$ & $\begin{array}{c}\text { CBZ+PHT } \\
\text { combitherap }\end{array}$ & PHT \\
monotherapy
\end{tabular}

Figure no. 1: Linear correlations between serum conc. and seizure frequency for carbamazepine.

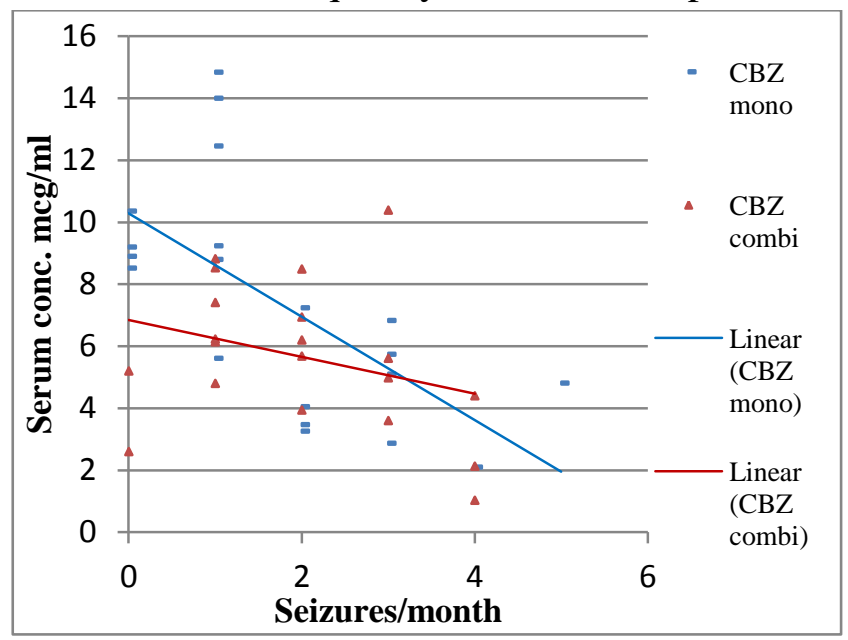

CBZ monotherapy: $y=-1.666 x+10.285 ; R^{2}=0.4091$

CBZ combitherapy: $y=-0.5943 x+6.8447 ; R^{2}=0.0985$

In combination group $55 \%$ patients showed therapeutic, $40 \%$ showed subtherapeutic and 5\% showed toxic concentrations. In phenytoin monotherapy group the serum concentrations weretherapeutic in $65 \%$, subtherapeutic in $15 \%$ and toxic in 20\%. Whilstphenytoin when given in combination with carbamazepine resulted in plasma concentration that was therapeutic in $50 \%$, subtherapeutic in $40 \%$ patients and $10 \%$ patients' values were in toxicrange.

Regarding idiosyncratic side effects, out of 40 patients on phenytoin, four had coarse facial appearance. Other side effects of phenytoin were as follows: six had nausea and vomiting, twelve had gingival hypertrophy, four had drowsiness and one had ataxia. While, out of 40 patients taking carbamazepine; five had nausea and vomiting, three had gingival hypertrophy, two had tremors, and one had ataxia.

Two patients taking phenytoin alone showed tremors as adverse effect even though their serum phenytoin concentration was in therapeutic range. Two patients taking carbamazepine alone complained of ataxia and vertigo had toxic levels of serum carbamazepine. In combination group, two patients showed signs of tremors had therapeutic levels of carbamazepine and phenytoin. One patient in the same group exhibited nystagmus had his 
phenytoin in therapeutic range whereas his carbamazepine was in toxic range.

In phenytoin alone group, out of thirteen patients twelve had convulsions and two had adverse drug reaction, despite therapeutic concentration. In the same group three out of four patients had convulsions despite toxic concentrations.In carbamazepine alone group, out of ten patients seven had convulsionsdespite therapeutic concentration. In the same group three out of four patients had convulsions despite toxic concentrations. Whereas in combination group, all six patients with therapeutic concentration gave history of convulsions. Two out of them had adverse drug reaction. So, it can be said that most of the patients who showed some evidence of side effects due to the antiepileptic drugs had normal serum levels of the antiepileptic drugs. Thus, it's possible that Indian population has different therapeutic range of antiepileptic drugs from that given in standard literature. But larger study needs to be done to confirm the results.

Figure no. 2: Linear correlations between serum conc. and seizure frequency for phenytoin.

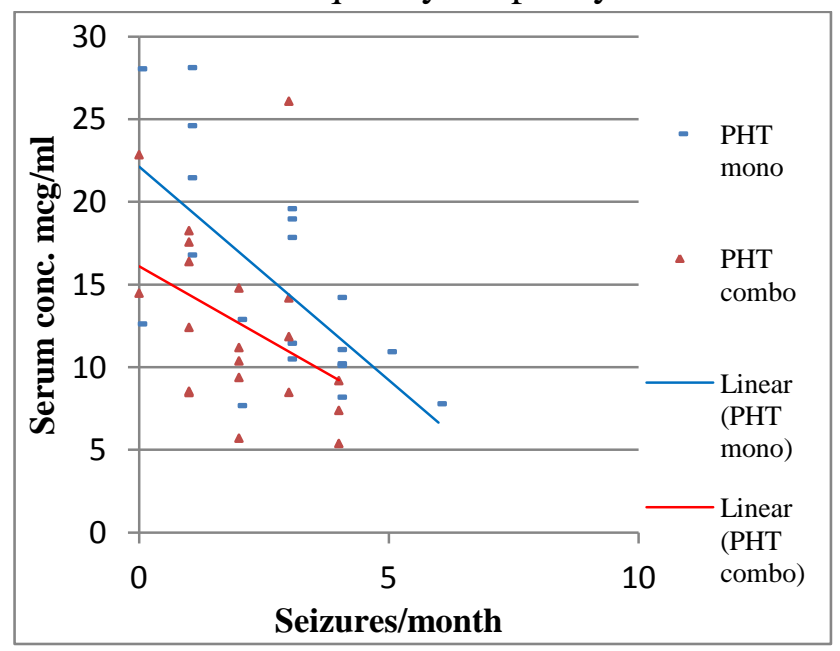

PHT monotherapy: $y=-2.5777 x+22.125 ; R^{2}=0.4344$ PHT combitherapy: $y=-1.7253 x+16.111 ; R^{2}=0.1552$

The study showed only 8 patients $(13.33 \%)$ became seizure-free during the study period while 52 patients $(86.67 \%)$ still experienced convulsions. There is a negative correlation between seizure frequency and serum concentrations for both the drugs, as depicted in Figure no. 1 and Figure no. 2.
Number of seizures reduced as serum level increased for both the drugs irrespective of given singly or in combination.

\section{DISCUSSION}

The statistical comparison of carbamazepine and phenytoin when given singly and in combination in a given population is determined mainly by the homogenicity of that population. In the present study, there were no significant differences in age, body weight, male/female ratio or sampling time among the three groups of patients.

In the present study, the time of sampling was standardized at $-12 \mathrm{~h}$ after the last carbamazepine and phenytoin dose, and patients had BD dosing schedule. Therefore, time interval between last dose and sample collection incarbamazepine and phenytoin levels probably did not contribute to any great extent to the observed variation. Patients with hepatic or renal disease were not included in this study to avoid the interference of drug protein binding and metabolism of the drug by these diseases.

Though majority of the patients were within the therapeutic range, all the patients did not show the same clinical outcome. Some patients can achieve therapeutic benefit at serum drug concentrations outside the reference ranges or at higher than routine doses.

Numerous studies demonstrated that carbamazepine metabolism is highly inducible by phenytoin and vice a versa. Johannessen and Strandjord, observed that patients receiving carbamazepine and phenytoin are required in larger doses when given together. Becausecarbamazepine concentration and carbamazepine level/dose ratio were lower than those in patients receiving carbamazepine alone. Wang et al., in their study found that clearance of carbamazepine was increased by phenytoin comedication. ${ }^{[14]}{ }^{[15]}$ Hua L and Delgado, 1995; had also studied interactions between phenytoin and carbamazepine. They observed that, patients receiving polytherapy were treated with larger carbamazepine doses than were patients treated with carbamazepine alone. Because the doses of 
carbamazepine in these patients were based on clinical response rather than serum carbamazepine level, this result may reflect the fact that these patients required a relatively larger carbamazepine dose than patients receiving carbamazepine monotherapy to maintain an adequate therapeutic effect. $^{[16]}$

Chapron et al.,in their study suggested- when epileptic patients receiving monotherapy require a second antiepileptic drug, and especially if comedication of phenytoin and carbamazepine is required, their larger doses may be needed to compensate for the increased elimination caused by the drug interactions. Chapron et al., on the other hand, enhance that in the process of tapering and discontinuing comedications, more frequent serum concentration monitoring is recommended during the ensuing deinducing phase to avoid sudden change in drug concentration and their toxicity. ${ }^{[17]}$ The main limitation of this study is that it is based on a small sample size of only 60 epileptic patients from one single tertiary care hospital. The study included those patients who were prescribed phenytoin and carbamazepine for treating epilepsy only. Thus, a study of a bigger magnitude would prove useful in the future.

\section{CONCLUSION}

We tried to systematically investigate the effect of carbamazepine and phenytoin comedication on their concentrations in the Indian population. Our results suggest that despite concentration being in subtherapeutic or toxic range, subjects may not necessarily have toxicity of failure. Therefore, we recommend that, though it is necessary to determine the serum levels of carbamazepine and phenytoin for dose adjustment and in avoidance of clinical problems owing to interactions of drug combinations, Dose titration should be done by correlating the serum drug levels with both; clinical outcome and the safety profile.

\section{ACKNOWLEDGEMENT}

We would like to thank the patients who participated in the study.
FUNDING: There was no funding source.

\section{CONFLICT OF INTEREST}

No conflict of interest.

\section{REFERENCES}

1. Santhosh NS, Sinha S, Satishchandra P. Epilepsy: Indian perspective. 2014; 17(March).

2. Reaction AD. Phenytoin and carbamazepine cross reactivity: report of a case and review of literature. 2003;703-4.

3. Lowenstein D. H. Seizures and EpilepsyFauci A.S, Braunwald E, Kasper D. L, Hauser S. L, Longo D. L, Jameson J. L, Loscalzo J. Harrison's Principles of Medicine. 17th edition.2008.

4. Brunton L. L, Chabner B. A, Knollmann B. C. Goodman \& Gilman's The Pharmacological Basis of Therapeutics. 12th Ed. Chapter 21;Pharmacotherapy of the Epilepsies, page 526582

5. Trost LF, Wender RC, Suter CC, Von Worley AM, Brixner DI, Rosenberg JH, Gunter MJ."Management of epilepsy in adults. Treatment guidelines". Postgraduate Medicine, December 2005, 118 (6): 29-33

6. C.L. Deckers, S.J. Czuczwar, Y.A. Hekster, A. Keyser, H. Kubova, H. Meinardi, et al.,Selection of antiepileptic drug polytherapy based on mechanisms of action: theevidence reviewed, Epilepsia 41 (2000) 1364-1374.

7. F. Bugamelli, C. Sabbioni, R. Mandrioli, E. Kenndler, F. Albani, M. Raggi, Simulta-neous analysis of six antiepileptic drugs and two selected metabolites in humanplasma by liquid chromatography after solid-phase extraction, Anal. Chim. Acta472 (2002) 1-10.

8. L. Budakova, H. Brozmanova, M. Grundmann, J. Fischer, Simultaneous determi-nation of antiepileptic drugs and their two active metabolites by HPLC, J. Sep.Sci. 31 (2008) 1-8.

9. K.M. Patil, S.L. Bodhankar, Simultaneous determination of lamotrigine,phenobarbitone, carbamazepine and phenytoin in human serum by high-performance liquid chromatography, J. Pharm. Biomed. Anal. 39 (2005)181-186. 
10. H.M. Neels, A.C. Sierens, K. Naelaerts, S.L. Scharpé, G.M. Hatfield, W.E. Lambert, Therapeutic drug monitoring of old and newer anti-epileptic drugs, Clin. Chem.Lab. Med. 42 (2004) 1228-1255.

11. P.N. Patsalos, W. Fröscher, F. Pisani, C.M. van Rijn, The importance of druginteractions in epilepsy therapy, Epilepsia 43 (2002) 365-385.

12. G.A. Khoschsorur, F. Frühwirth, G. HalwachsBaumann, Simple and rapid HPLCmethod for simultaneous determination of multiple antiepileptic drugs inhuman serum, Chromatographia 54 (2001) 345-349.

13. Ferreira A, Rodrigues M, Oliveira P, Francisco J, Fortuna A, Rosado L, et al. Liquid chromatographic assay based on microextraction by packed sorbent for therapeutic drug monitoring of carbamazepine, lamotrigine, oxcarbazepine, phenobarbital, phenytoin and the active metabolites carbamazepine-10,11epoxide and licarbazepine. J Chromatogr B Anal Technol Biomed Life Sci [Internet]. 2014;971:20-9. Available from: http://dx.doi.org/10.1016/j.jchromb.2014.09.010

14. Johannessen SI, Strandjord RE. The influence of phenobarbital and phenytoin on carbamazepine serum level. In: Schneider H, Janz D, GardnerThorpe C, Meinardi H, Sherwin AL, eds. Clinical pharmacology of anti-epileptic drug. Berlin: Springer-Verlag, 1975:201-5

15. Wang RD, Liu LT, Yiu CH, Chang TY. Carbamazepine drug interactions: the influence of concurrent drug therapy on serum concentrations of carbamazepine and its epoxide metabolite.Chin Med J (Taipei) 1990;45:222-32.

16. Liu H, Delgado MR, Browne RH. Interactions of valproic acid with carbamazepine and its metabolites' concentrations, concentrations ratios, and level/dose ratios in epileptic children. Clin Neuropharmacol [Internet]. 1995;18(1):112. Available from: http://www.ncbi.nlm.nih.gov/pubmed/866552

17. Chapron DJ, LaPierre BA, Abou-Elkair M. Unmasking the significant enzyme-inducing effects of phenytoin on serum carbamazepine concentrations during phenytoin withdrawal. Ann Pharmacother 1993;27:708-11. 